\title{
ABORDAGEM ALGÉBRICO-DIFERENCIAL APLICADA ÀS EQUAÇÕES DE BALANÇO PARA UM ESCOAMENTO BIFÁSICO LÍQUIDO-VAPOR
}

\author{
R. G. D. TEIXEIRA ${ }^{1}$, A. R. SECCHI ${ }^{2}$ e E. C. BISCAIA Jr ${ }^{2}$ \\ ${ }^{1}$ CENPES (Centro de Pesquisas e Desenvolvimento), PETROBRAS \\ ${ }^{2}$ Programa de Engenharia Química (PEQ), COPPE, UFRJ \\ E-mail para contato: rodrigogdt@ petrobras.com.br
}

\begin{abstract}
RESUMO - O escoamento bifásico líquido-vapor por tubulações ocorre com frequência nas indústrias do petróleo e de energia nuclear. Cálculos de projeto, simulação e otimização de tais unidades de processo exigem métodos precisos e numericamente eficientes para a previsão de variações de pressão e frações volumétricas das fases em tais dutos. Este trabalho aborda um escoamento bifásico representativo de refinarias de petróleo, em estado estacionário. O problema foi equacionado através do modelo de mistura denominado Drift-Flux Model, resultando em quatro equações diferenciais de balanço, acrescidas de equações algébricas constitutivas. Propõe-se, como alternativa mais eficiente que as consolidadas técnicas iterativas baseadas na discretização por volumes finitos, a solução do modelo por métodos indicados para Sistemas de Equações Algébrico-Diferenciais. Foram obtidos ganhos de eficiência e resultados mais acurados frente à técnica mais difundida, confirmando-se a superior eficácia da proposta.
\end{abstract}

\section{INTRODUÇÃO}

Escoamentos concorrentes de líquido e vapor por tubulações (ou, mais sucintamente, escoamentos bifásicos, como são designados neste trabalho) são encontrados com frequência na indústria de processos químicos, notadamente nos setores de petróleo e de energia nuclear, mas também se manifestando em sistemas de refrigeração (Xu e Fang, 2012) e até mesmo na indústria farmacêutica (Smith et al., 2012). A produção de petróleo, por exemplo, pode ser descrita como um escoamento bifásico gás-líquido, já que o mesmo é sempre extraído junto com quantidades variáveis de gás natural (Thomas, 2001). O escoamento desta produção por oleodutos ou gasodutos também está sujeito à ocorrência deste fenômeno (Mokhatab e Poe, 2012). Já em refinarias, antes de ser destilado, o petróleo é aquecido em um forno industrial, seguindo parcialmente vaporizado por uma linha de transferência para a torre fracionadora (Jones e Pujadó, 2006).

O projeto, simulação e otimização dos supracitados processos dependem de modelos e técnicas numéricas adequadas para a previsão de regimes de escoamento, variações de pressão e frações volumétricas das fases nos referidos dutos. Estas variáveis são influenciadas pela transferência de massa entre as fases líquida e vapor, que pode ocorrer simultaneamente ao escoamento, e também deve ser considerada (Brill e Mukherjee, 1999; Ishii e Hibiki, 2011). 


\section{REVISÃO BIBLIOGRÁFICA}

\subsection{Escoamento Monofásico}

Efetuando-se balanços de massa, momento e energia sobre um elemento infinitesimal de volume através do qual um fluido Newtoniano de interesse escoa, obtêm-se as três principais equações de balanço: as equações da continuidade, do movimento e da energia. São equações diferenciais parciais no domínio do tempo e nas três dimensões do espaço, as quais, complementadas com uma equação de estado e outra para a entalpia (além de condições iniciais e de contorno), determinam completamente as distribuições de pressão, massa específica, velocidade, entalpia e temperatura do escoamento (Bird et al., 2004).

As três equações de balanço aplicam-se, em sua forma original, a qualquer escoamento monofásico laminar. Quando o escoamento se torna turbulento (caracterizado por flutuações caóticas das velocidades, pressões e temperaturas em cada ponto do fluido), há diversas alternativas de solução a considerar. O método mais adotado parte do reconhecimento de que ao buscar-se a solução para dado problema turbulento, o que se deseja na verdade são os valores médios das diversas variáveis em torno dos quais ocorrem as flutuações caóticas irregulares. Matematicamente, este entendimento resulta na chamada decomposição de Reynolds: variáveis como a pressão, as componentes da velocidade e a temperatura são substituídas nas equações de balanço pela soma de seus valores médios com as correspondentes flutuações. Efetua-se em seguida a média temporal das relações obtidas, do que resultam as equações da continuidade, do movimento e da energia expressas em termos dos valores médios desejados. A primeira destas é inteiramente análoga à equação da continuidade original; as novas equações do movimento e da energia diferem das antigas apenas pelo surgimento das tensões de Reynolds e do fluxo térmico turbulento, cujas determinações dependem de modelagem adicional. Ishii e Hibiki (2011) comparam o procedimento de efetuação da média temporal à aplicação de um filtro passa-baixa, resultando na exclusão de flutuações de alta frequência indesejadas. Esta analogia é fundamental para a modelagem do escoamento bifásico, como se verá a seguir.

\subsection{Escoamento Bifásico Líquido-Vapor}

A característica visual mais marcante a distinguir o escoamento bifásico do monofásico é a distribuição física das fases pelo espaço da tubulação, a qual é denominada regime de escoamento. $\mathrm{O}$ regime denominado slug, por exemplo, caracteriza-se pela alternância periódica de grandes quantidades de líquido e gás (Brill e Mukherjee, 1999), e deve ser evitado no projeto e operação de unidades de processo, dado que cria dificuldades para os sistemas de controle, podendo provocar até mesmo danos físicos às instalações (Mokhatab e Poe, 2012; Nolte, 1979). O regime de escoamento vigente em dado problema varia com a orientação e com o diâmetro do tubo, e também com as vazões relativas das fases que escoam e suas propriedades físicas (Brill e Mukherjee, 1999; Mokhatab e Poe, 2012).

Do ponto de vista da modelagem matemática, a principal distinção deste escoamento frente ao monofásico dá-se pela presença de uma ou múltiplas interfaces a separar regiões ocupadas por 


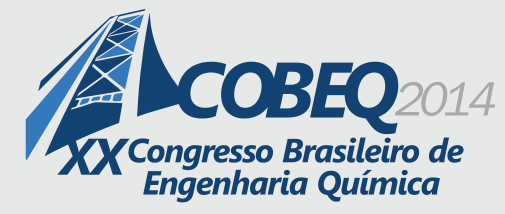

19 a 22 de outubro de 2014

Florianópolis/SC

somente uma das fases, no interior das quais as equações de balanço discutidas permanecem válidas. Mediante condições de contorno e tratamento adequado nas interfaces, chega-se a uma formulação válida do escoamento. Entretanto, a solução do modelo resultante - onde às turbulências de cada fase se somam a caótica deformação e movimento das interfaces - é dificultada (em muitos casos, impossibilitada) por dificuldades de natureza matemática e limitações de capacidade computacional. Trata-se de um obstáculo semelhante ao encontrado em escoamentos monofásicos turbulentos, o que sugere o emprego da mesma ferramenta - a média temporal - para sua mitigação (Ishii e Hibiki, 2011).

Uma alternativa, então, é buscar o cálculo do escoamento mediante a solução do conjunto completo de equações de balanço para cada fase (Two-Fluid Model). Outra possibilidade passa pelo chamado Drift-Flux Model (DFM), composto por três equações de balanço aplicadas à mistura, acrescidas de uma equação da continuidade para a fase vapor. Além de menor custo computacional (menos equações e menos incógnitas) e ampla aplicabilidade, a segunda opção oferece a vantagem de representar o comportamento global da mistura (e não de cada fase), que é o resultado de interesse na solução de problemas práticos de engenharia (Kleinstreuer, 2003; Ishii e Hibiki, 2011). Estas vantagens justificam a seleção, no presente trabalho, do modelo DFM, visando calcular um escoamento bifásico em estado estacionário. As equações foram resolvidas em sua forma unidimensional, tomando-se as médias das variáveis sobre a seção reta do tubo como valores representativos. Então, aplicando-se a média temporal e integrando-se o resultado sobre a área transversal $(A)$ da tubulação de diâmetro interno $d$, obtém-se finalmente:

$$
\begin{aligned}
& \frac{d\left(\rho_{m} v_{m}\right)}{d z}=0 \\
& \frac{d\left(\alpha_{v} \rho_{v} v_{m}\right)}{d z}=\Gamma_{v}-\frac{d}{d z}\left[\frac{\alpha_{v} \rho_{v} \rho_{l}}{\rho_{m}} v_{d}\right] \\
& \frac{d\left(\rho_{m} v_{m}^{2}\right)}{d z}=-\frac{d P}{d z}-\rho_{m} g \operatorname{sen}(\theta)-\rho_{m} \frac{f}{2 d} v_{m}\left|v_{m}\right|-\frac{d}{d z}\left[\frac{\alpha_{v} \rho_{v} \rho_{l}}{\left(1-\alpha_{v}\right) \rho_{m}} v_{d}^{2}\right] \\
& \frac{d\left(\rho_{m} h_{m} v_{m}\right)}{d z}=\frac{Q_{W} \xi}{A}-\frac{d}{d z}\left[\frac{\alpha_{v} \rho_{v} \rho_{l}}{\rho_{m}}\left(h_{v}-h_{l}\right) v_{d}\right]+\left[v_{m}+\frac{\alpha_{v}\left(\rho_{l}-\rho_{v}\right)}{\rho_{m}} v_{d}\right] \frac{d P}{d z}
\end{aligned}
$$

As Equações (1) - (4) são as equações do DFM para a continuidade da mistura, continuidade da fase vapor, movimento e energia da mistura, respectivamente. Talebi et al. (2012) obtiveram boa reprodução de dados experimentais utilizando este modelo, reforçando a validade das simplificações inerentes (desconsideração de tensões viscosas, da condução de calor axial, etc.). A coordenada axial é representada por $z$ e a inclinação da tubulação em relação à horizontal, por $\theta$ ( $g$ é a aceleração da gravidade). Velocidades, massas específicas, entalpias e a pressão são simbolizadas por $v, \rho, h$ e $P$. Os índices $m, l$ e $v$, por sua vez, denotam a mistura bifásica e as fases líquida e vapor. $Q_{W}$ representa o fluxo de calor trocado entre os fluidos que escoam e as vizinhanças da tubulação, sendo $\xi$ o perímetro interno do duto. A Equação 3 inclui um fator de atrito $f$, e o primeiro membro à direita na Equação $2\left(\Gamma_{v}\right)$ representa a transferência de massa entre as fases líquida e vapor (devido a mudanças de fase). A fração $\alpha_{v}$ surge na efetuação da média temporal e, embora coincida com a fração volumétrica local da fase vapor, seu significado físico rigoroso é a probabilidade de se encontrar esta 


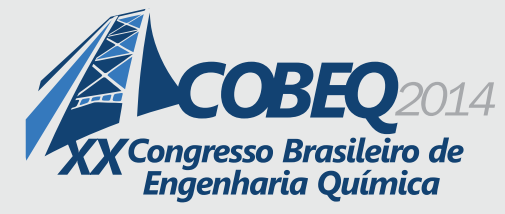

fase no ponto considerado. A massa específica da mistura e sua entalpia são definidas pelas Equações 5 e 6 (Kleinstreuer, 2003; Ishii e Hibiki, 2011):

$$
\begin{aligned}
& \rho_{m}=\left(1-\alpha_{v}\right) \rho_{l}+\alpha_{v} \rho_{v} \\
& h_{m}=\frac{\left(1-\alpha_{v}\right) \rho_{l} h_{l}+\alpha_{v} \rho_{v} h_{v}}{\rho_{m}}
\end{aligned}
$$

O movimento relativo entre as fases é introduzido neste modelo pela diferença entre as médias (sobre a área transversal) da velocidade da fase vapor e do fluxo volumétrico total (dado por $j=\left(Q_{l}+Q_{v}\right) / A$, onde $Q_{i}$ é a vazão volumétrica da fase $\left.i\right)$. Esta diferença é a definição da velocidade média de arrasto $v_{d}$ (tradução de mean drift velocity), para a qual dispõe-se da seguinte relação constitutiva:

$$
v_{d}=\frac{\rho_{m}\left[\widetilde{v_{d}}+\left(C_{0}-1\right) v_{m}\right]}{\rho_{m}-\left(C_{0}-1\right)\left(\rho_{l}-\rho_{v}\right) \alpha_{v}}
$$

$\mathrm{Na}$ Equação 7, o coeficiente de distribuição $C_{0}$ introduz o efeito das variações de $\alpha_{v}$ e $j$ sobre a seção reta do escoamento, enquanto $\widetilde{v_{d}}$ é a média sobre a área $A$, ponderada por $\alpha_{v}$, da velocidade local de arrasto (Kleinstreuer, 2003; Ishii e Hibiki, 2011). Ambos são determinados por relações adicionais, disponíveis para cada regime de escoamento.

\section{OBJETIVO}

A literatura recente é rica em textos que praticam a discretização das equações do escoamento bifásico em volumes finitos, seguindo-se sua solução por métodos iterativos semi-implícitos como o conhecido algoritmo SIMPLE e seus derivados (Patankar, 1980). Uma vez que se baseiam na solução sequencial das equações do modelo, tais métodos são classificados como segregados (Prosperetti e Tryggvason, 2007; Talebi et al., 2012). Observa-se, todavia, que o modelo DFM em sua forma unidimensional estacionária consiste em um Problema de Valor Inicial formado por quatro Equações Diferenciais Ordinárias em que variáveis como as propriedades físicas e a velocidade média de arrasto devem obedecer a determinadas restrições. Isto permite classificar a formulação apresentada como um Sistema de Equações Algébrico-Diferenciais, ou EAD (Kunkel e Mehrmann, 2006). Este reconhecimento, por si só, já constitui um avanço na medida que sugere a possibilidade de solução do problema por técnicas numéricas desenvolvidas especificamente para este tipo de sistema.

Segundo Brenan et al. (1996), entre as primeiras técnicas utilizadas com sucesso na solução de sistemas EAD, encontra-se o método BDF (Backward Differentiation Formulas). Além de ser um método multipasso, proporcionando mais alta ordem e acurácia, esta abordagem apresenta a importante vantagem de solucionar todas as equações (diferenciais e restrições) do problema de forma simultânea, do que se pode esperar economia de esforço computacional frente às supracitadas técnicas segregadas iterativas para o modelo em questão. Assim, o presente trabalho tem como objetivo demonstrar a superior eficiência da abordagem algébrico-diferencial para o modelo DFM unidimensional estacionário frente aos métodos segregados consolidados, utilizando para tanto o código DASSLC (que faz uso do método BDF). 


\section{METODOLOGIA}

Foi considerado neste trabalho um escoamento hipotético através de uma tubulação horizontal, exposta ao ar ambiente (temperatura ambiente $T_{S U R}=27^{\circ} \mathrm{C}$ ), com diâmetro interno de 7,981 in e 12 metros de comprimento. $\mathrm{O}$ fluxo de calor recebido pelo escoamento vale:

$$
Q_{W}=U\left(T_{S U R}-T\right)
$$

$\mathrm{Na}$ Equação 8, T representa a temperatura do escoamento, e o coeficiente global de transferência de calor $U$ é dado por:

$$
U=\frac{2}{d}\left[\frac{\ln (D / d)}{k}+\frac{2}{D h_{o}}\right]^{-1}
$$

$\mathrm{Na}$ Equação 9, $D$ e $k$ representam o diâmetro externo da tubulação $(8,625$ in) e a condutividade térmica de sua composição (aço carbono, $k=60,5 \mathrm{~W} / \mathrm{m} / \mathrm{K}$ ). O coeficiente $h_{o}$, referente à convecção de calor na parede externa da tubulação, foi calculado pela correlação para convecção forçada de Churchill e Bernstein (1977), adotando-se uma velocidade de vento de $36 \mathrm{~km} / \mathrm{h}$. Foram consideradas negligenciáveis as diferenças de temperatura entre o escoamento e a parede interna da tubulação, justificando a não-inclusão da correspondente resistência convectiva na Equação 9.

Os cálculos das propriedades físicas das fases exigem informação sobre suas composições químicas. Visando obter resultados para um cenário típico de processos de refino de petróleo, pouco explorados na literatura relacionada, supôs-se um escoamento de $115000 \mathrm{~kg} / \mathrm{h}$ de nafta (fração separada em refinarias; base da gasolina), com composição global dada pela Tabela 1. As composições das fases líquida e vapor foram determinadas na entrada da tubulação $\left(P=4,95 \mathrm{kgf} / \mathrm{cm}^{2}\right.$ e $T=163,95{ }^{\circ} \mathrm{C}$ ) e nos demais pontos mediante cálculos de Equilíbrio Líquido-Vapor, adotando-se sempre a equação de estado de Peng-Robinson. Similarmente, as variações de todas as propriedades físicas com $T$ e $P$ foram computadas por meio de métodos e correlações apresentados por Riazi (2005) e Daubert e Danner (1997) para frações de petróleo de composição química definida.

Em todos os cálculos realizados, o escoamento bifásico permanece no regime denominado "Bolhas" (Bubble), o que foi assegurado por meio do mapa de Mandhane et al. (1974) para tubos horizontais. Neste regime, Brill e Mukherjee (1999) recomendam o cálculo do fator de atrito $f$ a partir do conhecido diagrama de Moody, adotando-se um número de Reynolds dado por $R e=\rho_{m} v_{m} d / \mu_{m}$, onde a viscosidade $\mu_{m}$ é calculada de forma análoga à Equação 5 . Neste mesmo regime, $C_{0}$ e $\widetilde{v_{d}}$ podem ser obtidos pelas Equações 10 e 11, onde $\sigma$ representa a tensão superficial (Levy, 1999; Ishii e Hibiki, 2011):

$$
\begin{aligned}
& C_{0}=1,2-0,2 \sqrt{\frac{\rho_{v}}{\rho_{l}}} \\
& \widetilde{v_{d}}=1,53\left(\sqrt[4]{\frac{\sigma g\left(\rho_{l}-\rho_{v}\right)}{\rho_{l}^{2}}}\right)
\end{aligned}
$$


A transferência interfacial de massa $\Gamma_{v}$ foi determinada de acordo com a Equação 12:

$$
\Gamma_{v}=\frac{Q_{W} \xi}{A} \frac{1}{h_{v}-h_{l}}
$$

Todos os cálculos comparativos foram implementados no MATLAB (o código DASSLC, desenvolvido em linguagem C, foi utilizado em uma DLL). A discretização das Equações (1) - (4) em volumes finitos foi realizada seguindo-se a metodologia geral apresentada por Patankar (1980) e Versteeg e Malalasekera (2007). Em acordo com a metodologia de malhas deslocadas (staggered grids), as velocidades foram calculadas nas faces dos volumes e as demais variáveis, em seus centros. A técnica upwind foi aplicada a todos os termos convectivos, favorecendo a convergência dos procedimentos iterativos. Desta forma, cada equação de balanço do modelo DFM deu origem a um sistema tridiagonal de equações, os quais foram todos resolvidos pelo algoritmo de Thomas, também conhecido como TDMA (Tri-Diagonal Matrix Algorithm). O algoritmo SIMPLER foi selecionado para a solução iterativa sequencial do problema. Ao fim de cada iteração deste método, foram atualizados os valores das propriedades físicas das fases e da mistura nos centros dos volumes finitos (as temperaturas também foram atualizadas nesta etapa, sendo determinadas a partir da correspondente entalpia da mistura e da Equação 6 pela função $f z e r o$ do MATLAB).

Tabela 1 - Composição molar da nafta

\begin{tabular}{cc}
\hline Componente & Porcentagem molar $(\%)$ \\
\hline$n$-Pentano & 4,03 \\
$n$-Heptano & 13,55 \\
2-Metil-Heptano & 16,98 \\
Ciclohexano & 17,28 \\
Benzeno & 3,72 \\
Tolueno & 25,26 \\
Etilbenzeno & 4,57 \\
$p$-Xileno & 10,96 \\
$o$-Xileno & 3,65 \\
\hline
\end{tabular}

\section{RESULTADOS E DISCUSSÃO}

O solver DASSLC necessitou de apenas 1,03 segundos para resolver o problema com tolerâncias absoluta e relativa de $10^{-9}$ e $10^{-6}$. Já o algoritmo SIMPLER, com 25 volumes, demorou 9,58 s. Com 50,100 e 200 volumes, este método levou 27,15 s, 56,75 s e 439,01 s, respectivamente.

A Figura 1 ilustra os principais resultados obtidos. Além dos perfis axiais de pressão e fração de vapor, são apresentados também os erros relativos, ponto a ponto, desta última grandeza (em relação à solução do DASSLC). Observa-se aí que somente com 200 volumes é que o erro relativo da fração de vapor em todos os pontos cai abaixo de $0,10 \%$. 

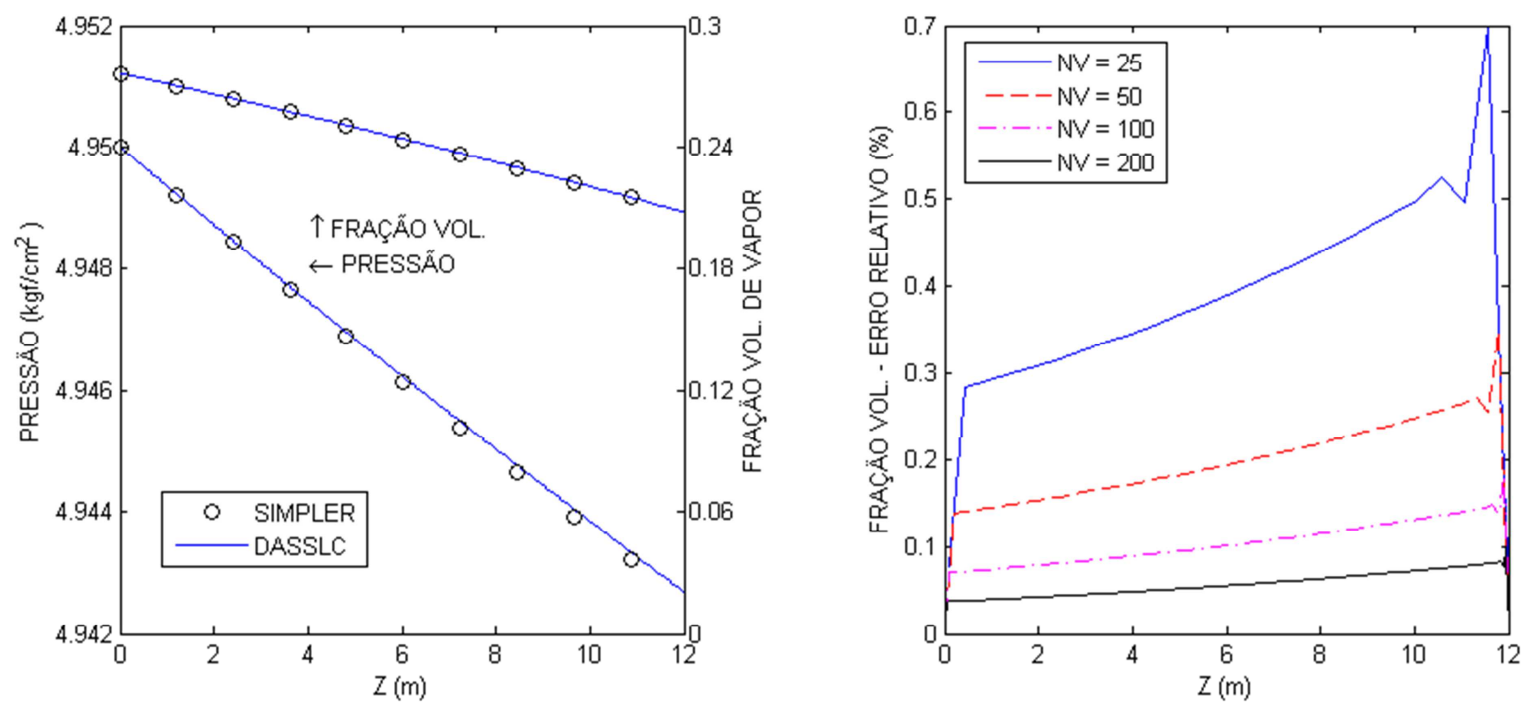

Figura 1 - (a) Perfis de pressão e fração de vapor calculados com o código DASSLC e com o algoritmo SIMPLER (200 volumes); (b) Erros relativos das frações de vapor calculadas com o algoritmo SIMPLER para diversos NV (Número de Volumes).

O segundo gráfico mostra que o comportamento geral da discretização em volumes finitos assemelha-se ao de um método de primeira ordem, dado que os erros em cada ponto se mostram diretamente proporcionais ao tamanho do "passo" (isto é, do volume). A baixa ordem desta técnica explica sua baixa acurácia, exigindo-se o emprego de um número elevado de volumes na busca por melhores resultados. Este fator, juntamente com a solução segregada das equações, torna este método muito ineficiente em comparação com a abordagem algébrico-diferencial, na qual são empregados passos variáveis e o método BDF de alta ordem (quarta ou quinta).

\section{CONCLUSÃO}

Este trabalho demonstrou a aplicação de uma metodologia concisa, porém completa, para o cálculo de diversas quantidades de interesse em escoamentos bifásicos por tubulações. A abordagem algébrico-diferencial se mostrou mais eficiente e acurada que o consolidado algoritmo SIMPLER para a solução do presente problema, conforme a análise teórica apresentada permitia esperar.

\section{REFERÊNCIAS}

BIRD, R. B.; STEWART, W. E.; LIGHTFOOT, E. N. Fenômenos de Transporte. Rio de Janeiro: Editora LTC - Livros Técnicos e Científicos, 2004.

BRENAN, K. E.; CAMPBELL, S. L.; PETZOLD, L. R. Numerical Solution of Initial-Value Problems in Differential-Algebraic Equations. Philadelphia: Editora Society for Industrial and Applied Mathematics, 1996. 


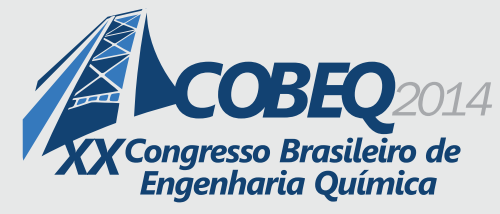

19 a 22 de outubro de 2014

Florianópolis/SC

BRILL, J. P.; MUKHERJEE, H. Multiphase Flow in Wells. Richardson: Society of Petroleum Engineers, 1999.

CHURCHILL, S. W.; BERNSTEIN, M. A Correlating Equation for Forced Convection From Gases and Liquids to a Circular Cylinder in Crossflow. J. Heat Transfer, v. 99, p. 300-306, 1977.

DAUBERT, T. E.; DANNER, R. P. API Technical Data Book - Petroleum Refining. Washington D.C.: American Petroleum Institute, 1997.

ISHII, M.; HIBIKI, T. Thermo-Fluid Dynamics of Two-Phase Flow. New York: Editora Springer, 2011.

JONES, D. S. J. S.; PUJADÓ, P. R. Handbook of Petroleum Processing. Dordrecht: Editora Springer, 2006.

KLEINSTREUER, C. Two-Phase Flow: Theory and Applications. New York: Editora Taylor \& Francis, 2003.

KUNKEL, P.; MEHRMANN, V. Differential-Algebraic Equations - Analysis and Numerical Solution. Zürich: Editora European Mathematical Society, 2006.

LEVY, S. Two-Phase Flow in Complex Systems. New York: Editora John Wiley \& Sons, 1999.

MANDHANE, J. M.; GREGORY, G. A.; AZIZ, K. A Flow Pattern Map for Gas-Liquid Flow in Horizontal Pipes. Int. J. Multiphase Flow, v. 1, p. 537-553, 1974.

MOKHATAB, S.; POE, W. A. Handbook of Natural Gas Transmission and Processing. Waltham: Editora Gulf Professional Publishing, 2012.

NOLTE, C. B. Optimum Pipe Size Selection. Houston: Editora Gulf Publishing Company, 1979.

PATANKAR, S. V. Numerical Heat Transfer and Fluid Flow. New York: Editora McGraw-Hill, 1980.

PROSPERETTI, A.; TRYGGVASON, G. Computational Methods for Multiphase Flow. Cambridge: Editora Cambridge University Press, 2007.

RIAZI, M. R. Characterization and Properties of Petroleum Fractions. West Conshohocken: American Society for Testing and Materials, 2005.

SMITH, T. R.; SCHLEGEL, J. P.; HIBIKI, T.; ISHII, M. Two-phase flow structure in large diameter pipes. Int. J. Heat Fluid Fl., v. 33, p. 156-167, 2012.

TALEBI, S.; KAZEMINEJAD, H.; DAVILU, H. A numerical technique for analysis of transient twophase flow in a vertical tube using the Drift-Flux Model. Nucl Eng Des, v. 242, p. 316-322, 2012.

THOMAS, J. E. Fundamentos de Engenharia de Petróleo. Rio de Janeiro: Editora Interciência, 2001.

VERSTEEG, H. K.; MALALASEKERA, W. An Introduction to Computational Fluid Dynamics. Harlow: Editora Pearson Education, 2007.

$\mathrm{XU}, \mathrm{Y}$; FANG, X. A new correlation of two-phase frictional pressure drop for evaporating flow in pipes. Int. J. Refrig., v. 35, p. 2039-2050, 2012. 